\title{
Relativistic Motion with Viscosity: II Stokes's Law of Resistance
}

\author{
Lorenzo Zaninetti \\ Physics Department, Turin, Italy \\ Email:1.zaninetti@alice.it
}

How to cite this paper: Zaninetti, L. (2021) Relativistic Motion with Viscosity: II Stokes's Law of Resistance. International Journal of Astronomy and Astrophysics, 11, 481-488.

https://doi.org/10.4236/ijaa.2021.114025

Received: September 15, 2021

Accepted: November 12, 2021

Published: November 15, 2021

Copyright $\odot 2021$ by author(s) and Scientific Research Publishing Inc. This work is licensed under the Creative Commons Attribution International License (CC BY 4.0).

http://creativecommons.org/licenses/by/4.0/

(c) (i) Open Access

\begin{abstract}
The deduction of a relativistic and mildly relativistic equation of motion in the presence of a drag force proportional to the velocity is presented. The obtained results are used to model the trajectory of the supernova SN1993J and the light curves of gamma-ray bursts.
\end{abstract}

\section{Keywords}

Supernovae, General Supernovae, Individual (SN 1993J) ISM, Supernova

Remnants GRB, Individual (GRB 130427A) GRB, Individual (GRB 120521C)

GRB, Individual (GRB 130606A)

\section{Introduction}

A relativistic treatment of the equation of motion in the presence of a resistive force proportional to the velocity has been investigated in the following models: a model for the Newtonian scattering of photons [1], a motion through a uniform adiabatic medium on the steady-state accretion of matter onto a Schwarzschild black hole [2], an extreme mass-ratio inspirals around strongly accreting supermassive black holes [3], and ultra-relativistic detonations in the framework of the cosmological first-order phase transitions [4]. In Section 2, this paper explores the relativistic law of motion in the presence of viscosity proportional to the velocity. Section 3 is devoted to the astrophysical applications.

\section{The Equation of Motion}

\subsection{The Classic Case}

We assume a one-dimensional motion with a resistive force of Stokes type [5], $F_{r e s}=-A m v(t)$, where $A$ is a constant, $m$ is the considered mass and $v(t)$ is the velocity. The differential equation which governs the motion is 


$$
v(t)=\frac{v_{0} \mathrm{e}^{-A t}}{\mathrm{e}^{-t_{0} A}},
$$

which has an analytical solution in an explicit form

$$
v\left(t ; A, v_{0}, t_{0}\right)=v(t)=\frac{v_{0} \mathrm{e}^{-A t}}{\mathrm{e}^{-t_{0} A}},
$$

where $v_{0}$ is the velocity at $t=t_{0}$. The equation of motion in the explicit form is

$$
r\left(t ; A, v_{0}, t_{0}, r_{0}\right)=-\frac{v_{0}\left(\mathrm{e}^{-A t}-\mathrm{e}^{-t_{0} A}\right) \mathrm{e}^{t_{0} A}}{A}+t_{0}
$$

where $r_{0}$ is the distance at $t=t_{0}$. The numerical value of the constant $A$ is

$$
A=\frac{\ln \left(\frac{v_{1}}{v_{0}}\right)}{t_{0}-t_{1}},
$$

where $v_{1}$ is the velocity at $t=t_{1}$.

\subsection{The Relativistic Case}

We assume a one-dimensional motion with a resistive force of Stokes type, $F_{r e s}=-A m_{0} v(t)$, where $A$ is a constant, $m_{0}$ is the considered rest mass and $v(t)$ is the velocity. Newton's second law in special relativity is:

$$
F=\frac{\mathrm{d}}{\mathrm{d} t}\left(\frac{m_{0} v(t)}{\sqrt{1-\frac{v(t)^{2}}{c^{2}}}}\right),
$$

where $F$ is the force, $m_{0}$ is the rest mass, $c$ is the velocity of light and $v(t)$ is the velocity; see Equation (7.16) in [6]. The first order differential equation in the velocity which governs the relativistic motion is

$$
\frac{\frac{\mathrm{d}}{\mathrm{d} t} v(t)}{\left(1-\frac{(v(t))^{2}}{c^{2}}\right)^{\frac{3}{2}}}=-A(v(t)) \text {. }
$$

An analytical solution to the above first order differential does not exist; however, a solution exists for $v(t)$ in an implicit form for the time

$$
\begin{gathered}
t=\frac{N}{D}, \\
N=\left(-2 c^{3}+2 c v_{0}^{2}\right) \sqrt{c^{2}-v^{2}}-\left(\left(-2 t_{0} A+\ln \left(\sqrt{c^{2}-v^{2}}-c\right)\right.\right. \\
\left.-\ln \left(c+\sqrt{c^{2}-v^{2}}\right)-\ln \left(\sqrt{c^{2}-v_{0}^{2}}-c\right)+\ln \left(c+\sqrt{c^{2}-v_{0}^{2}}\right)\right) c^{2} \\
-2 c \sqrt{c^{2}-v_{0}^{2}}-v_{0}^{2}\left(-2 t_{0} A+\ln \left(\sqrt{c^{2}-v^{2}}-c\right)-\ln \left(c+\sqrt{c^{2}-v^{2}}\right)\right. \\
\left.\left.-\ln \left(\sqrt{c^{2}-v_{0}^{2}}-c\right)+\ln \left(c+\sqrt{c^{2} v_{0}^{2}}\right)\right)\right)(c-v)(c+v) .
\end{gathered}
$$


and

$$
D=2 A\left(c^{2}-v^{2}\right)\left(c^{2}-v_{0}^{2}\right)
$$

where $v_{0}$ is the velocity at $t=t_{0}$. The constant $A$ can be derived from the following formula

$$
A=\frac{N N}{D D}
$$

where

$$
\begin{aligned}
N N= & \left(-2 c^{3}+2 c v_{1}^{2}\right) \sqrt{c^{2}-v_{0}^{2}}+\left(c+v_{0}\right)\left(c-v_{0}\right)\left(\left(\ln \left(\sqrt{c^{2}-v_{1}^{2}}-c\right)\right.\right. \\
& \left.-\ln \left(c+\sqrt{c^{2}-v_{1}^{2}}\right)-\ln \left(\sqrt{c^{2}-v_{0}^{2}}-c\right)+\ln \left(c+\sqrt{c^{2}-v_{0}^{2}}\right)\right) c^{2} \\
& +2 \sqrt{c^{2}-v_{1}^{2}} c-v_{1}^{2}\left(\ln \left(\sqrt{c^{2}-v_{1}^{2}}-c\right)-\ln \left(c+\sqrt{c^{2}-v_{1}^{2}}\right)\right. \\
& \left.\left.-\ln \left(\sqrt{c^{2}-v_{0}^{2}}-c\right)+\ln \left(c+\sqrt{c^{2}-v_{0}^{2}}\right)\right)\right),
\end{aligned}
$$

and

$$
D D=2\left(t_{0}-t_{1}\right)\left(c^{2}-v_{1}^{2}\right)\left(c^{2}-v_{0}^{2}\right),
$$

where $v_{1}$ is the velocity at $t=t_{1}$.

\subsection{The Mildly-Relativistic Case}

The first order differential equation for the mildly-relativistic motion is

$$
\frac{\mathrm{d}}{\mathrm{d} t} v(t)+\frac{3 v(t)^{2}\left(\frac{\mathrm{d}}{\mathrm{d} t} v(t)\right)}{2 c^{2}}=-A v(t),
$$

which has solution

$$
v\left(t ; t_{0}, v_{0}\right)=\mathrm{e}^{-A t-\frac{\left(\frac{3}{2 c^{2}\left(\mathrm{e}^{B}\right)^{2}\left(\mathrm{e}^{A t}\right)^{2}}\right)}{2}-B},
$$

where $W$ is the Lambert $\mathrm{W}$ function [7] and

$$
B=-\frac{4 t_{0} A c^{2}+4 \ln \left(v_{0}\right) c^{2}+3 v_{0}^{2}}{4 c^{2}},
$$

with $v_{0}$ being the velocity at $t=t_{0}$. The trajectory in the mildly relativistic case is

$$
r\left(t ; t_{0}, r_{0}, v_{0}\right)=-\frac{\mathrm{e}^{t_{0} A} c^{2} v_{0}(W(D)+3)\left(\mathrm{e}^{\frac{v_{0}^{2}}{c^{2}}}\right)^{\frac{3}{4}}-3 \mathrm{e}^{A t} \sqrt{\mathrm{e}^{W(D)}}\left(\left(A r_{0}+v_{0}\right) c^{2}+\frac{v_{0}^{3}}{2}\right)}{3 \sqrt{\mathrm{e}^{W(D)}} \mathrm{e}^{A t} c^{2} A},
$$

where 


$$
D=\frac{3 v_{0}^{2} \mathrm{e}}{2 c^{2}},
$$

with $r_{0}$ being $r$ at $t=t_{0}$. The constant $A$ can be derived in the mildly relativistic case by the following formula

$$
A\left(t_{0}, t_{1}, v_{0}\right)=\frac{-v_{0}^{2}\left(4 \ln \left(\frac{v_{0}}{v_{1}}\right) c^{2}+3 v_{0}^{2}-3 v_{1}^{2}\right)}{4 v_{0}^{2} c^{2}\left(t_{0}-t_{1}\right)},
$$

where $v_{1}$ is the velocity at $t=t_{1}$.

\subsection{Astrophysical Luminosity}

The mechanical relativistic luminosity is

$$
L_{m, r}=4 \pi r(t)^{2} \frac{1}{1-\beta(t)^{2}} \rho_{0}\left(\frac{r_{0}}{r}\right)^{d} c^{3} \beta(t),
$$

where $r(t)$ is the temporary radius of the expansion, $r_{0}$ is the radius at $t=t_{0}, \rho_{0}$ is the density at $t=t_{0}, d$ is a shape parameter and $\beta(t)=\frac{v(t)}{c}$. The observed luminosity, $L_{\text {obs }}$, is assumed to scale as

$$
L_{o b s}=C_{o b s} L_{m, r}\left(1-\mathrm{e}^{-\tau_{v}}\right),
$$

where $C_{o b s}$ is a constant that allows the match between theory and observations, and $-\tau_{v}$ is the optical thickness.

\section{Astrophysical Applications}

The astrophysical units are chosen to be $\mathrm{pc}$ for the length and years for the time: the constant $A$ is therefore expressed in $\frac{1}{\mathrm{yr}}$. A test for the quality of the fits is represented by the merit function $\chi^{2}$

$$
\chi^{2}=\sum_{j} \frac{\left(r_{t h}-r_{o b s}\right)^{2}}{\sigma_{o b s}^{2}}
$$

where $r_{t h}, r_{o b s}$ and $\sigma_{o b s}$ are the theoretical radius, the observed radius and the observed uncertainty, respectively.

\subsection{Application to SN 1993J}

Figure 1 reports the numerical trajectory, of SN 1993J for which observational parameters are available [8] [9] with data as in Table 1.

\subsection{Application to GRBs}

A first example is applied to the light curve (LC) of GRB 130427A, which was the most luminous gamma-ray burst in the last 30 years; see Figure 1 in [10]. Figure 2 reports the $\mathrm{X}$-flux as a function of the time and the relative theoretical data, with data as in Table 2. 


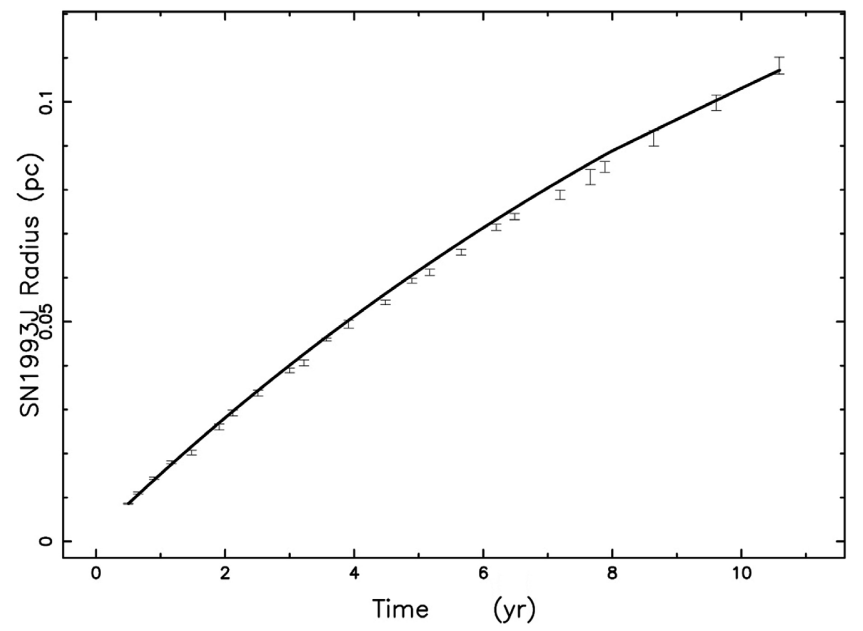

Figure 1. Numerical radius (full line) and astronomical data of SN 1993J with vertical error bars.

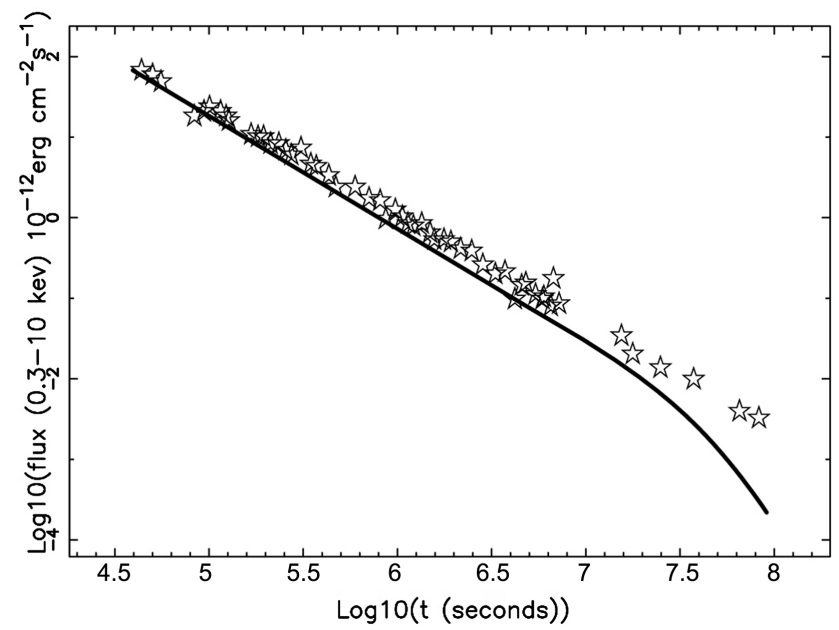

Figure 2. Flux in the X-ray as a function of time in seconds for GRB 130427A (empty stars) and theoretical curve as given by Equation (20) (full line) when $\tau_{v}=\infty$ with data are as in Table 2.

Table 1. Numerical values for the parameters of Stokes's theoretical model applied to SN 1993J.

\begin{tabular}{ccc}
\hline model & values & $\chi^{2}$ \\
\hline Stokes's & $r_{0}=3.0 \times 10^{-3} \mathrm{pc} ; v_{0}=13800 \mathrm{~km} / \mathrm{s} ; A=0.07 \frac{1}{\text { years }}$ & 85.7 \\
\hline
\end{tabular}

A second example is applied to the LC in X-ray of GRB 120521C 2, see Figure 2 in [11], which is reported in Figure 3, with temporal behavior of the optical depth as in Figure 4.

A third example is given by the LC in X-ray of GRB 130606A, see Figure 2 in [11], which is reported in Figure 5, with the temporal behavior of the optical depth as in Figure 6. 


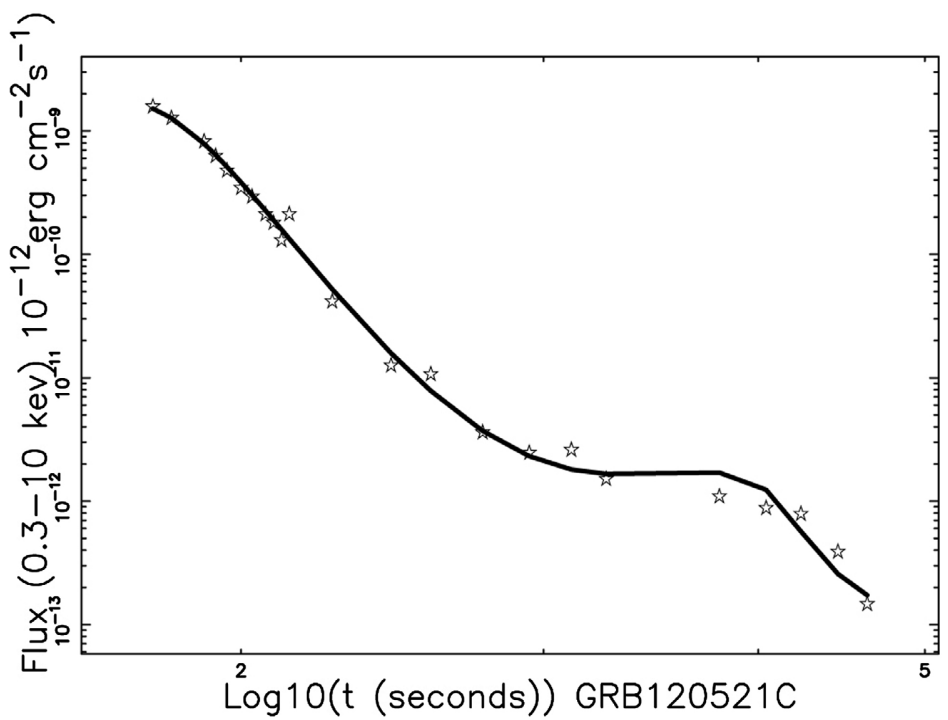

Figure 3. Flux in the X-ray as function of time in seconds for GRB 120521C (empty stars) and theoretical curve as given by Equation (20) (full line), with $\tau_{v}$ as in Figure 4 and with data as in Table 2.

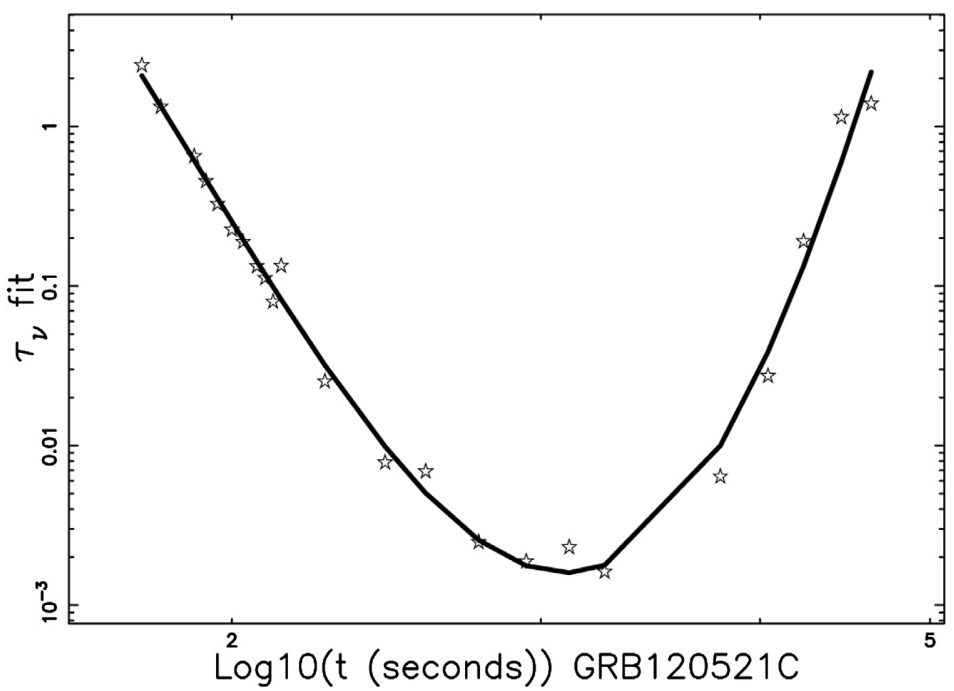

Figure 4. The time dependence of $\tau_{v}$ (empty stars) for GRB 120521C and a logarithmic polynomial approximation of degree 5 (full line). Parameters as in Table 2.

Table 2. Numerical values of the parameters for the theoretical model.

\begin{tabular}{cc}
\hline GRB name & theoretical parameters \\
\hline $\begin{array}{c}\text { GRB } \\
130427 \mathrm{~A}\end{array}$ & $r_{0}=9.9 \times 10^{-5} \mathrm{pc} ; t_{0}=1.0 \times 10^{-3}$ year $; \beta_{0}=0.9 ; A=1 \frac{1}{\mathrm{pc}} ; d=3.1$ \\
GRB & $r_{0}=1.0 \times 10^{-4} \mathrm{pc} ; t_{0}=1.0 \times 10^{-6}$ year $; \beta_{0}=0.9 ; A=10000 \frac{1}{\mathrm{pc}} ; d=3$ \\
$120521 \mathrm{C}$ & \\
GRB & $r_{0}=1.0 \times 10^{-4} \mathrm{pc} ; t_{0}=1.0 \times 10^{-6}$ year $; \beta_{0}=0.9 ; A=1000 \frac{1}{\mathrm{pc}} ; d=2$ \\
$130606 \mathrm{~A}$ &
\end{tabular}




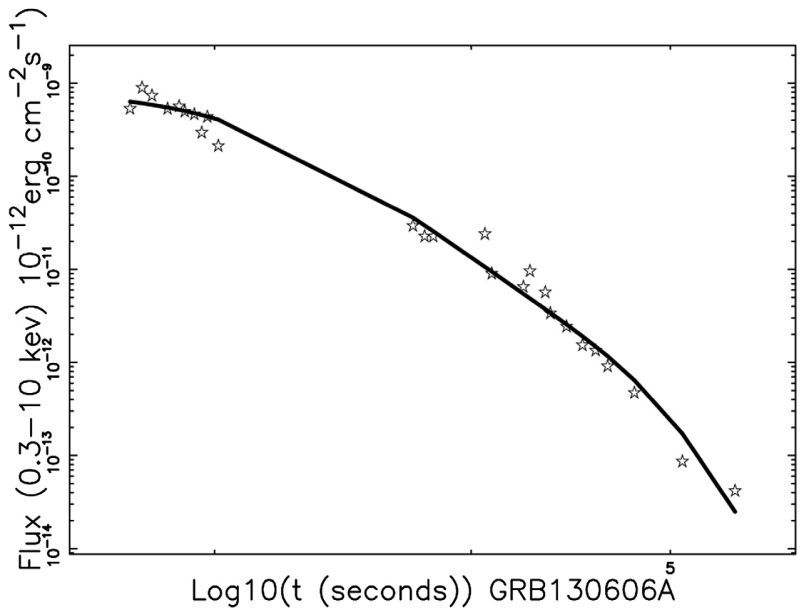

Figure 5. Flux in the X-ray as a function of time in seconds for GRB 130606A (empty stars) and theoretical curve as given by Equation (20) (full line), with $\tau_{v}$ as in Figure 6 and with data as in Table 2.

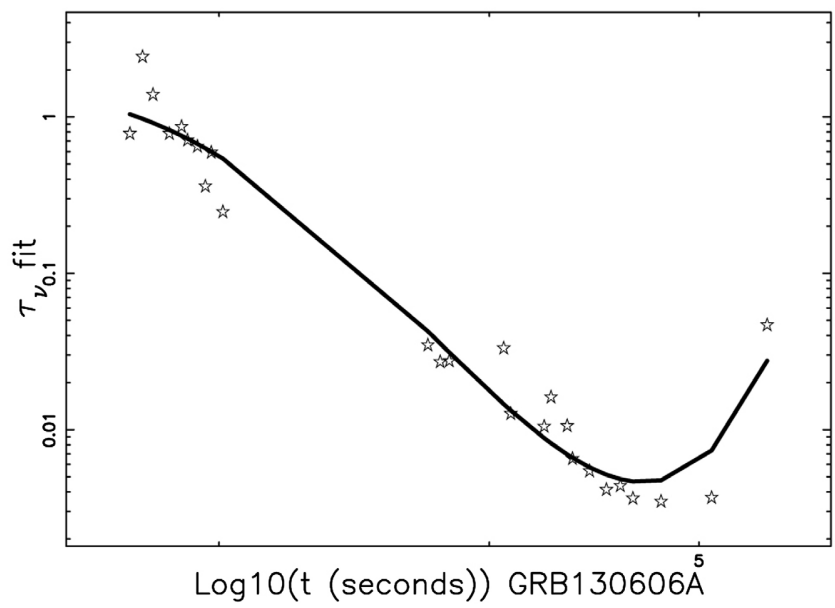

Figure 6. The time dependence of $\tau_{v}$ (empty stars) for GRB 130606A and a logarithmic polynomial approximation of degree 5 (full line). Parameters as in Table 2.

\section{Conclusions}

We analyzed the one-dimensional relativistic motion in the presence of a resistive force proportional to the velocity. An analytical solution for the velocity was derived in an implicit form, see Equation (7). In the mildly relativistic case, we derived an analytical solution for both the velocity, see Equation (14), and the distance, see Equation (16), in terms of the Lambert W function.

A first test to evaluate the constant $A$ in an astrophysical environment is on SN 1993J. A full relativistic treatment of the LC for GRBs was done for GRB 130427A, GRB 120521C and GRB 130606A in the framework of the optical thickness with a time dependence.

\section{Conflicts of Interest}

The author declares no conflicts of interest regarding the publication of this paper. 


\section{References}

[1] Syer, D. (1994) Relativistic Dynamical Friction in the Weak Scattering Limit. Monthly Notices of the Royal Astronomical Society, 270, 205-208. https://doi.org/10.1093/mnras/270.1.205

[2] Petrich, L.I., Shapiro, S.L., Stark, R.F. and Teukolsky, S.A. (1989) Accretion onto a Moving Black Hole: A Fully Relativistic Treatment. Astrophysical Journal, 336, p. 313. https://doi.org/10.1086/167013

[3] Barausse, E. (2007) Relativistic Dynamical Friction in a Collisional Fluid. Monthly Notices of the Royal Astronomical Society, 382, 826-834. https://doi.org/10.1111/j.1365-2966.2007.12408.x

[4] Leitao, L. and Mégevand, A. (2016) Hydrodynamics of Ultra-Relativistic Bubble Walls. Nuclear Physics B, 905, 45-72. https://doi.org/10.1016/j.nuclphysb.2016.02.009

[5] Stokes, G.G., et al. (1851) On the Effect of the Internal Friction of Fluids on the Motion of Pendulums. Pitt Press, Cambridge.

[6] French, A.P. (1968) Special Relativity. CRC, New York.

[7] Lambert, J.H. (1758) Observations variae in Mathesin Puram. Acta Helvitica, physico-mathematico-anatomico-botanico-medica, 3, 128.

[8] Marcaide, J.M., Mart-Vidal, I., Alberdi, A., Pérez-Torres, M.A., et al. (2009) A Decade of SN 1993J: Discovery of Radio Wavelength Effects in the Expansion Rate. $A s$ tronomy \& Astrophysics, 505, 927-945. https://doi.org/10.1051/0004-6361/200912133

[9] Mart-Vidal, I., Marcaide, J.M., Alberdi, A., Guirado, J.C., Pérez-Torres, M.A. and Ros, E. (2011) Radio Emission of SN1993J: The Complete Picture. II. Simultaneous Fit of Expansion and Radio Light Curves. Astronomy \& Astrophysics, 526, Article No. A143. https://doi.org/10.1051/0004-6361/201014517

[10] De Pasquale, M., Page, M., Kann, D., Oates, S., Schulze, S., Zhang, B., Cano, Z., Gendre, B., Malesani, D., Rossi, A., Gehrels, N., Troja, E., Piro, L., Boër, M. and Stratta, G. (2017) Challenging the Forward Shock Model with the 80 Ms Follow up of the X-Ray Afterglow of Gamma-Ray Burst 130427A. Galaxies, 5, 6. https://doi.org/10.3390/galaxies5010006

[11] Yasuda, T., Urata, Y., Enomoto, J. and Tashiro, M.S. (2017) Hard X-Ray Spectral Investigations of Gamma-Ray Bursts 120521C and 130606A at High-Redshift z $\sim 6$. Monthly Notices of the Royal Astronomical Society, 466, 4558-4567. https://doi.org/10.1093/mnras/stw3130 